\title{
Usability Lab
}

\author{
Michael B. Twidale \\ Graduate School of Library and Information Science \\ University of Illinois \\ USA \\ twidale@illinois.edu
}

\section{INTRODUCTION}

Carefully designed rigorous experimental protocols run in controlled settings are necessary for certain types of empirical scientific research. However there are cheaper, faster, more lightweight approaches that are good enough to fit the grubby pragmatics of iterative engineering design - trying to make things a bit better one step at a time. This usability lab aims to show how user tests can be designed to fit this particular need.

It is possible to find a few of the more glaring usability problems with a website or application in a very short time. Even a user test with one person can give some indicators of potential problems meriting more detailed analysis. It is definitely more informative than no user test at all - which is what, unfortunately, often happens in design iterations.

\section{WHAT HAPPENS}

The lab will be run in an informal, entertaining manner, following the format of a gameshow. We ask for submissions in advance for websites or applications to be analysed (email the author). Someone involved in the application design needs to be present at the lab to help us understand the designers' aims and if there are particular aspects of the design or its use that they want insights into. We ask for a volunteer who is unfamiliar with the application/website to go out of the room. In 10 minutes we come up with some representative tasks that are relevant and of interest to the application designers. We then invite the volunteer back into the room and conduct a 10 minute user study "live in front of a studio audience". Then we spend 10 minutes on analysis and design recommendations, involving the audience in what they have seen.

This approach is deliberately designed with education and entertainment in mind. It is intended to help audience members who have never seen or participated in a user study to get some flavour of what they are or how they are conducted, and how analysis may be done.

Of course, actually running a user study this way would be silly. 10 minutes? In front of an audience? With one subject? However, even doing considerable violence to the idealised user study method, we have found that with careful control of the process and appropriate kinds of analysis, we almost always find something useful to say about the application under study.

Participants will get a sense of the power of carefully watching people use an application, asking follow-up questions, and analyzing what happened and why in the light of theories of Human Computer Interaction and a substantial body of empirical studies and prior experience.

That is not to say that this minimalist approach finds all or even most of the usability problems. It obviously doesn't. However can find some rather glaringly obvious ones, but even these may not be the most important. We are not saying: "now try exactly this at home". But we are saying: "now you've seen what can be done under the performative constraints of this workshop, why don't you try something like a proper user test at home? You don't need a lot of subjects, a user test lab or even a white coat. Just have a go. You might well find something important very quickly".

\section{REFERENCES}

Marty, P. \& Twidale, M. (2004). Lost in gallery space: A conceptual framework for analyzing the usability flaws of museum websites. First Monday, 9(9).

Twidale, M. \& Marty, P. (2005). Come On Down! A game show approach to illustrating usability evaluation methods. interactions 12(6) 24-27.

Marty, P. \& Twidale, M. (2005). Usability@90mph: Presenting and Evaluating a New, High-Speed Method for Demonstrating User Testing in Front of an Audience. First Monday, 10(7). 